\section{Isolation of the Bound Form of Nicotinic Acid}

Chaudhuri and Kodicek ${ }^{1}$ have shown that almost the whole of the nicotinic acid in cereal bran was present in a bound form. They concentrated this 'precursor' of nicotinic acid from wheat bran considerably. Chaudhuri ${ }^{2}$, starting from rice bran in this laboratory, concentrated this substance about seventy times, following the concentration by the usual colorimetric method using $p$-aminobenzoic acid as the coupling reagent ${ }^{3}$. It has now been possible to isolate the bound form of nicotinic acid from rice bran in a crystalline state. This work has been facilitated by the observation that the active substance, when subjected to paper chromatography using phenol-watel $(80: 20)$ as the solvent system, gave two ninhydrinsensitive spots, one of which moved with the solvent front and produced a blackish-violet colour with ninhydrin. It also gave an orange colour when sprayed with $p$-aminobenzoic acid after exposure to cyanogen bromide vapour according to the method of Kodicek and Reddi'. The orange colour has been shown to be produced by the bound form, and it is insoluble in amyl alcohol, which dissolves the colour produced by free nicotinic acid as observed by Swaminathan ${ }^{5}$. A method developed by Das and Ghosh $^{6}$ based on this observation has been used to follow the concentration of the bound form of nicotinic acid in the present work.

The method of isolation consists of : (1) the extraction of rice bran with $0.1 \mathrm{~N}$ hydrochloric acid; (2) precipitating inactive material from the extract with 3 volumes of acetone; (3) centrifuging and precipitating the active material from the centrifugate with 3 volumes of a mixture of acetone and ether (2:1); (4) extracting the precipitate with 65 per cent alcohol and precipitating the active material from the extract with 12 vol. of acetone-ether $(2: 1)$ and repeating this process; (5) drying the precipitate, dissolving it in the minimum quantity of water, fractionating it on a cellulose column by treating the column with phenol-water $(80: 20)$ when the active material forming the brown zone moves with the solvent front, and collecting the brown phenol-water fraction; (6) precipitating the active substance from the phenol-water solution with 45 vol. of acetoneether $(2: 1)$ in the cold ; (7) washing the precipitate with acetone-ether, drying, dissolving it in the minimum quantity of cold water, and allowing it to crystallize in a vacuum desiccator in the cold.

The substance crystallizes in shining russet rectangular plates (Fig. 1). Several preparations showed the same crystalline structure, composition and activity. The yield is 50-70 $\mathrm{mgm}$. per $100 \mathrm{gm}$. of rice bran. It decomposes above $225^{\circ} \mathrm{C}$. It gives a blue fluorescence in ultra-violet light, which changes to yellowish-green on treatment with alkali, as was observed with their wheat-bran concentrate by Chaudhuri and Kodicek ${ }^{1}$. Dissolved in $0.1 \mathrm{~N}$ hydrochloric acid, it gives an absorption maximum at $262 \mathrm{m \mu}$. Analysis of the substance gives the following figures: $\mathrm{C}, 47.41 ; \mathrm{H}, 6.93 ; \mathrm{N}, 11.65 ; \mathrm{S}, 0.85$ per cent. Molecular weight, as determined by the diffusion technique using a sintered 'Pyrex' glass crucible fitted with a fritted disk (grade $G-3$ ), has been found to be of the order of 7,000, which agrees fairly well with the theoretical molecular weight $(7,200)$ calculated on the assumption that there is on $\theta$ molecule of nicotinic acid per molecule of the sub-

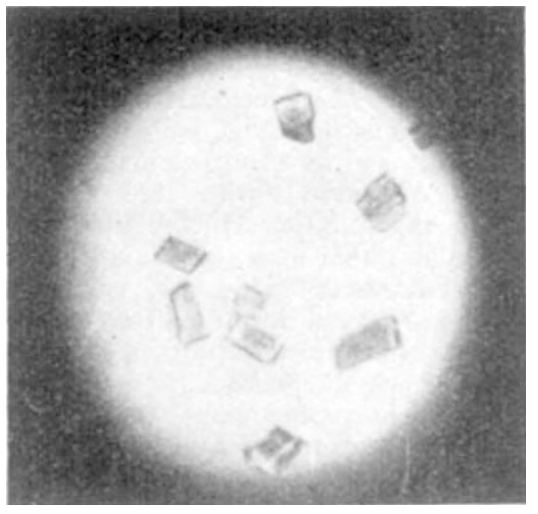

H'ig. 1. Crystals of the bound form of nicotinic acid. $(\times 8)$

stance. Preliminary experiments indicate that it is a peptide bound with a nicotinic acid moiety.

In future communications this bound form of nicotinic acid, from which nicotinic acid is liberated by mild alkali treatment, will be referred to as 'niacinogen' for convenience.

We thank Dr. J. J. Ghosh, Dr. N. C. Ghosh and Dr. S. Ghosh for their kind assistance and also the Indian Council of Medical Research for financing this work.

B. C. GUHA

M. L. DAS

Department of Applied Chemistry,

University College of Science and Technology, Calcutta. July 22.

1 Chaudhuri, D. K., and Kodicek, E., Nature, 165, 1022 (1950).

2 Chaudhuri, D. K., Science and Culture, 19, 409 (1954).

'Chaudhuri, D. K., Ind. J. Med. Res., 39, 491 (1951).

- Kodicek, E., and Reddi, K. K., Nature, 168, 475 (1951).

3 Swaminathan, N., Nature, 141, 830 (1938).

- Das, M. I., and Ghosh, N. C., Ind. J. Med. Res. (in the press).

\section{Chromatographic Characterization of Soluble Deoxypolynucleotides released in Mouse Spleen by X-Irradiation}

IN connexion with a programme of studies concerning the biochemical effects of whole-body X-irradiation on the deoxyribonucleic acid-protein complex of radio-sensitive tissues of the mouse, we wished to explore the nature of the soluble deoxypolynucleo. tides released in the spleen after exposure to the irradiation.

Previously reported evidence ${ }^{1}$ indicates that follow ing whole-body $\mathrm{X}$-irradiation of mice at the $L D 100$ dose-level (810-850 r.) there occurs a significant rise in free deoxypolynucleotide values from a pre-expos. ure level of $20 \mathrm{\mu gm}$. per spleen to a maximum of $350 \mu \mathrm{gm}$. per spleen (average value) 4-6 hr. postexposure. This maximum value occurs at the same post-exposure time when the spleen weight is decreasing and the deoxyribonucleic acid-nucleoprotein content of the spleen is falling precipitously. These deoxypolynucleotides were characterized by their solubility in $0 \cdot 14 M$ sodium chloride solution (deoxyribonucleic acid-nucleoprotein is insoluble in $0.14 \mathrm{M}$ saline), insolubility in cold $0 \cdot 2 M$ perchloric acid, ultra-violet absorption characteristics, positive Dische 